\title{
Vanishing left ventricular thrombus in a woman with peripartum cardiomyopathy: a case report
}

\author{
Wafa A Altuwaijri', lain DC Kirkpatrick ${ }^{2}$, Davinder S Jassal ${ }^{2,3,4,5^{*}}$ and Anita Soni ${ }^{4}$
}

\begin{abstract}
Background: Peripartum cardiomyopathy (PPCM) is a rare cardiac disorder characterized by the development of heart failure in the last month of pregnancy or up to 5 months postpartum in women without other identifiable causes of cardiac failure. The combination of left ventricular (LV) systolic dysfunction and hypercoaguability can cause thromboembolic complications including intra-cardiac thrombi.
\end{abstract}

Case presentation: A 25-year-old Caucasian female with PPCM demonstrated multiple thrombi in the LV on transthoracic echocardiography. Following anticoagulation with parenteral heparin, a cardiac MRI four days later demonstrated near resolution of the thrombi.

Conclusion: We review the presentation, diagnosis and management of LV thrombi in the clinical setting of PPCM.

Keywords: Peripartum cardiomyopathy, Multimodality cardiac imaging, Thrombus

\section{Background}

Peripartum cardiomyopathy (PPCM) is a rare cardiac disorder characterized by the development of heart failure in the last month of pregnancy or up to 5 months postpartum in women without other identifiable causes of cardiac failure. The combination of left ventricular (LV) systolic dysfunction and hypercoaguability can cause thromboembolic complications including intracardiac thrombi. Although there are two case reports of PPCM with rapid resolution of intracardiac thrombi diagnosed by transthoracic echocardiography (TTE) in the literature to date, our case illustrates the use of multimodality cardiac imaging including TTE and cardiac MRI (CMR) in this patient population. We review the presentation, diagnosis and management of LV thrombi in the clinical setting of PPCM.

\section{Case presentation}

A 25-year-old G4, P4 Caucasian female presented with a history of worsening dyspnea, orthopnea and paroxysmal nocturnal dyspnea seven months post-partum. On physical examination, she was tachycardic with a heart rate

\footnotetext{
* Correspondence: djassal@sbgh.mb.ca

${ }^{2}$ Department of Radiology, St. Boniface General Hospital, University of Manitoba, Winnipeg, Manitoba, Canada

${ }^{3}$ Section of Cardiology, Department of Internal Medicine, Faculty of Medicine, University of Manitoba, 409 Taché Avenue, Winnipeg R2H 2A6, Manitoba, Canada

Full list of author information is available at the end of the article
}

of $110 \mathrm{bpm}$ and a normal blood pressure of 110/ $70 \mathrm{mmHg}$. The heart sounds were within normal limits with decreased breath sounds bilaterally and lower limb edema. Initial blood work including the complete blood count, electrolytes, liver function tests and cardiac biomarkers were within normal limits. The electocardiogram demonstrated sinus tachycardia and the chest $\mathrm{x}$-ray confirmed cardiomegaly with pulmonary venous congestion. Transthoracic echocardiography (TTE) demonstrated biventricular dilation, biatrial enlargement, global hypokinesis of the left ventricle (LV) with an ejection fraction less than $20 \%$. There were multiple echodense masses within the LV, consistent with thrombi (Figure 1A). With a diagnosis of peripartum cardiomyopathy (PPCM), the patient was appropriately started on diuretics, beta blockade, ACE inhibition and parenteral heparin for the LV thrombi. A subsequent cardiac MRI (CMR) 4 days later confirmed severe biventricular systolic dysfunction, with significant resolution of the thrombi previously detected on TTE (Figure 1B).

\section{Discussion}

Peripartum cardiomyopathy (PPCM) is a disorder of unknown cause in which LV ventricular systolic dysfunction and symptoms of heart failure occur between the last month of pregnancy and the first 5 months postpartum. The incidence of PPCM varies from the infrequent one case per 4000 live births in the US to the more 


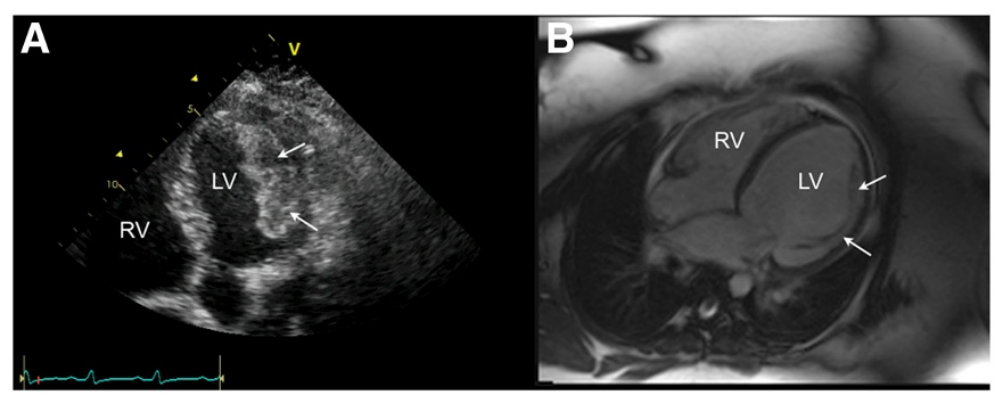

Figure 1 A) An apical 5 chamber view on TTE demonstrating a large layered echodense mass attached to the lateral wall of the LV consistent with thrombi. B) Four-chamber view of a balanced steady-state free precession CMR acquisition demonstrating that the thrombus has nearly completely resolved, leaving behind only the underlying muscular trabeculations on this view (arrows).

frequent one case per 1000 live births in South Africa or one case per 299 live births in Haiti [1]. Peripartum cardiomyopathy has been associated with several risk factors including increased age, gravidity or parity, African origin, toxaemia or hypertension of pregnancy, use of tocolytics, twin pregnancy, obesity and low socioeconomic status [1]. A number of potential etiologies for PPCM have been proposed including myocarditis, abnormal immune response to pregnancy, maladaptive response to the hemodynamic stresses of pregnancy, stress-activated cytokines, and prolonged tocolysis [1]. As a diagnosis of exclusion, women with PPCM are treated with similar medications as the general heart failure population including beta blockers, nitrates, hydralazine and digoxin [1].

Women with PPCM are at high risk for thrombus formation and thromboembolism due to both the hypercoagulable state of pregnancy and stasis of blood due to severe LV systolic dysfunction. Although twodimensional TTE is the most commonly used technique for the non-invasive identification and follow-up of LV thrombus, the recent introduction of contrast echo and cardiac MRI (CMR) may improve thrombus detection. In a recent study evaluating 361 patients, 2D TTE was compared to both TEE and CMR for the identification of LVT. Although TTE had a sensitivity and specificity of $23 \pm 12 \%$ and $96 \pm 4 \%$ for the diagnosis of LVT, CMR had a higher sensitivity and specificity of $88 \pm 9 \%$ and 99 $\pm 2 \%$, respectively [2]. In a different study, although the administration of LV opacification during TTE doubled sensitivity and improved accuracy for the detection of LVT, 9 out of 23 (39\%) thrombi were still missed as compared to delayed enhancement CMR imaging [3].

In women with PPCM, LV thrombus (LVT) is detected in up to $17 \%$ of patients, in whom the risk of systemic embolization is high [4]. A review of the literature demonstrated only two echocardiographic cases of rapid resolution of LVT during PPCM following parenteral anticoagulation [5,6]. Kim DY et al. described a 22-yearold female with a PPCM and multiple biventricular thrombi on TTE that resolved following 3 weeks of anticoagulation [5]. Nishi I et al. described a similar case of a 23-year-old with PPCM and biventricular thrombi on TTE that resolved rapidly after 4 days of parenteral anticoagulation [6]. Our case illustrates the use of multimodality cardiac imaging including TTE and CMR for both the detection and rapid resolution of LVT in this patient population. We recommend a minimum of 6 months of anticoagulation to prevent life threatening embolization in women with PPCM.

\section{Conclusion}

The use of multimodality cardiac imaging including TTE and CMR can provide complementary information for the management of LVT in the clinical setting of a PPCM.

\section{Consent}

Written informed consent was obtained from the patient for publication of this case report and accompanying images. A copy of the written consent is available for review by the Editor-in-Chief of this journal.

\section{Competing interests}

The authors declare that they have no competing interests.

\section{Authors' contributions}

WA, DJ, IK and AS contributed to the writing of the manuscript. All authors read and approved the final manuscript.

\section{Author details}

${ }^{1}$ Department of Internal Medicine, University of Manitoba, Winnipeg, Manitoba, Canada. ${ }^{2}$ Department of Radiology, St. Boniface General Hospital, University of Manitoba, Winnipeg, Manitoba, Canada. ${ }^{3}$ Section of Cardiology, Department of Internal Medicine, Faculty of Medicine, University of Manitoba, 409 Taché Avenue, Winnipeg R2H 2A6, Manitoba, Canada. ${ }^{4}$ Institute of Cardiovascular Sciences, Department of Physiology, St. Boniface General Hospital, University of Manitoba, Winnipeg, Manitoba, Canada. ${ }^{5}$ Cardiology, Radiology and Physiology, Bergen Cardiac Care Centre, St. Boniface General Hospital, Winnipeg R2H 2A6, Canada.

Received: 20 August 2012 Accepted: 1 October 2012 Published: 2 October 2012 


\section{References}

1. Bhattacharyya A, Basra SS, Sen P, Kar B: Peripartum cardiomyopathy: a review. Tex Heart Inst J 2012, 39(1):8-16.

2. Srichai MB, Junor C, Rodriguez LL, Stillman AE, Grimm RA, Lieber ML, Weaver JA, Smedira NG, White RD: Clinical imaging, and pathological characteristics of left ventricular thrombus: a comparison of contrastenhanced magnetic resonance imaging, transthoracic echocardiography, and transesophageal echocardiography with surgical or pathological validation. Am Heart J 2006, 152(1):75-84.

3. Weinsaft JW, Kim RJ, Ross M, Krauser D, Manoushagian S, LaBounty TM, Cham MD, Min JK, Healy K, Wang Y, Parker M, Roman MJ, Devereux RB: Contrast-enhanced anatomic imaging as compared to contrastenhanced tissue characterization for detection of left ventricular thrombus. JACC CardiovasC Imaging 2009, 2(8):969-79.

4. Amos AM, Jaber WA, Russell SD: Improved outcomes in peripartum cardiomyopathy with contemporary. Am Heart J 2006, 152(3):509-13.

5. Kim DY, Islam S, Mondal NT, Mussell F, Rauchholz M: Biventricular thrombi associated with peripartum cardiomyopathy. J Health Popul Nutr 2011, 29(2):178-80.

6. Nishi I, Ishimitsu T, Ishizu T, Ueno Y, Suzuki A, Seo Y, Ohtsuka S, lida K, Yamaguchi l: Peripartum cardiomyopathy and biventricular thrombi. Circ J 2002, 66(9):863-5.

doi:10.1186/1756-0500-5-544

Cite this article as: Altuwaijri et al:: Vanishing left ventricular thrombus in a woman with peripartum cardiomyopathy: a case report. BMC

Research Notes 2012 5:544.

\section{Submit your next manuscript to BioMed Central and take full advantage of:}

- Convenient online submission

- Thorough peer review

- No space constraints or color figure charges

- Immediate publication on acceptance

- Inclusion in PubMed, CAS, Scopus and Google Scholar

- Research which is freely available for redistribution 